\title{
The Influence of Masked Stimuli on Response Selection: Evidence from a Semantic Categorization Task
}

\author{
Brenda Ocampo ${ }^{1}$
}

Published online: 9 November 2016

(C) The Psychonomic Society, Inc. 2016

\begin{abstract}
Unconscious visual stimuli can be processed by human observers and influence their behaviour. A striking example is a phenomenon known as "free-choice priming," where masked "prime" stimuli- of which participants are unaware - modulate which of two response alternatives they are likely to choose. Recent efforts to uncover the mechanisms underlying this intriguing effect have revealed that free-choice priming can emerge even in the absence of automatized stimulus-response (S-R) associations between masked primes and specific motor responses, indicating that free choices can be influenced by a masked prime's meaning (Ocampo, 2015). It remains unknown, however, whether masked primes bias response selections because they are implicitly classified according to task instructions, or because spreading activation occurs within the prime's semantic network. To adjudicate between these two possibilities, participants in the present experiment categorised targets as either animals or people and selected which of two response alternatives they wanted to make following presentation of a free-choice target. Crucially, while implicit classifications could proceed during processing of both animal and person masked primes, only animal primes could trigger spreading activation within their semantic network. This manipulation modulated free-choice priming; only masked animal primes influenced response selections to free-choice targets. This result indicates that an automatic spreading activation mechanism might underlie a masked prime's ability to influence free-choice responses.
\end{abstract}

Brenda Ocampo

b.ocampo@psy.uq.edu.au

1 School of Psychology, The University of Queensland, St Lucia, Australia
Keywords Masked priming · Unconscious · Free-choice · Response selection · Semantic categorization

\section{Introduction}

The masked priming paradigm was first introduced by Forster and Davis (1984) to explore lexical processing under subliminal conditions. The three-step procedure involved the brief presentation of a prime word preceded by a forward mask (typically a series of \#s) and backward-masked by an uppercase target. Outside the psycholinguistics literature, the masked priming method has been used extensively to examine subliminal perception (see Klapp, 2015 for a review). In a typical experiment, participants are asked to classify the direction of a target arrow pointing either to the left or to the right. Targets are preceded by a prime arrow that is either congruent (pointing in the same direction as the target) or incongruent (pointing in the opposite direction). A mask presented immediately before and/or after the prime prevents its conscious detection. Nonconscious processing of the masked prime is established when it produces faster reaction times (and lower error rates) to a subsequent target that is congruent versus incongruent. A common aspect of most masked priming studies is that participants classify targets by making fixed responses that are either correct or incorrect; the only correct response to a left arrow target, for instance, is a left button press. Recently, researchers have begun to explore whether masked primes can influence responses that are not fixed as in the examples above, but free. Numerous studies have now demonstrated priming of free-choice responses (Bodner \& Mulji, 2010; Mattler \& Palmer, 2012; Kiesel et al., 2006; Klapp \& Hinkley, 2002; Schlaghecken \& Eimer, 2004), but the mechanisms underlying this effect remain unclear. The present study was designed to shed light on this matter by 
exploring whether free-choice priming in a semantic categorisation task can be better explained by implicit classification versus automatic spreading of activation accounts of priming.

\section{Free-choice priming}

In free-choice priming experiments, masked primes are sometimes followed by a free-choice target (e.g., " $<>$ ") to which participants are asked to respond by choosing which of two button-press responses they want to make. This manipulation produces a dramatic result: prime-congruent responses (e.g., a left button-press following a left arrow prime) are chosen more frequently and performed faster than primeincongruent responses (e.g., a left button-press following a right arrow prime), indicating that masked primes influenced participants' free-choice decisions (Bodner \& Mulji, 2010; Mattler \& Palmer, 2012; Kiesel et al., 2006; Klapp \& Hinkley, 2002; Schlaghecken \& Eimer, 2004). Several studies have demonstrated that free-choice priming is highly contextdependent; the effect does not arise, for example, when masked primes and visible targets correspond in meaning but do not share physical properties (e.g., $<<$ prime followed by LL target) or when fixed and free-choice trials are blocked rather than intermixed (Klapp \& Haas, 2005; Schlaghecken \& Eimer, 2004). It was suggested that a masked prime's influence on free-choice responses is limited to situations where prime stimuli are experienced as visible targets for which direct S-R links have been automatized and are continuously reinforced.

However, free-choice priming has recently been shown to emerge even in the absence of automatized S-R associations. Ocampo (2015) adapted the number classification task originally employed by Naccache and Dehaene (2001) to demonstrate priming by novel, "unseen" primes. This time, however, participants categorised target numbers 1, 4, 6, and 9 as less than or greater than 5, and made free-choice responses to a randomly intermixed free-choice target (\#). Half of the masked number primes appeared as targets during the experiment, whereas the other half (2, 3, 7 and 8$)$ did not. While S-R bonds were established for the former group of "repeat" primes, masked "novel" primes were never associated with an overt motor response and were thus assumed to require semantic-level processing to influence target-elicited responses. Consistent with the previous findings of Naccache \& Dehaene (2001), both repeat and novel primes influenced target-elicited responses. Specifically, target numbers were categorized faster (and more accurately) when preceded by masked primes belonging to the same category (e.g., a visible target " 9 " preceded by a masked prime " 6 ") versus the opposite category (e.g., a visible target "9" preceded by masked prime " 4 "). The new finding was that both types of primes influenced responses on free-choice trials, where participants selected the prime-congruent response $54 \%$ of the time (i.e., more often that would be expected by chance). There also was a trend towards faster RTs when free-choice selections were congruent versus incongruent with the masked prime, but the size of this effect was small $(\sim 8 \mathrm{~ms})$ and only trended towards significance $(p=0.06)$. Critically, because neither fixed nor free-choice priming effects were altered by whether or not masked primes appeared as targets, it was concluded that both types of priming might be attributed to comprehension of the masked prime's meaning.

In sum, the mechanisms underlying free-choice priming appear to manifest either at the response-level (via direct S$\mathrm{R}$ links) or at "deeper" levels of stimulus processing that involve semantic comprehension. Yet exactly how novel primes bias free-choice behaviour remains obscure. In fact, at least three different models can account for the findings of Ocampo (2015). Implicit classification, action trigger, and spreading activation theories will be considered in turn below.

\section{Priming by novel primes: Implicit classifications, action triggers, or spreading activation?}

Implicit classification theories propose that the same semantic categorisation procedures applied to conscious targets are applied to masked primes. Following semantic categorization masked primes activate their category-assigned response, producing response facilitation when subsequent targets require the same response and interference when they require a different response (Dehaene et al., 1998; Reynvoet, Gevers, \& Caessens, 2005). In Ocampo (2015) all masked primes were subjected to implicit classifications (i.e., is the number less than or greater than 5?), resulting in the activation of the category-assigned response even when primes were never seen as targets. An alternative explanation is provided by Kiesel, Kunde, and Hoffmann (2007), who proposed that masked primes can trigger responses as long as they fit existing action release conditions termed "action triggers." For instance, when participants were instructed to classify target numbers as less than or greater than 5, they prepared by linking all numbers less than 5 with a "less than" response and all numbers greater than 5 with a "greater than" response. In this way, novel primes become associated with their corresponding responses without being experienced as targets and importantly, without being subjected to semantic-level analyses. However, subsequent studies employed stimulus categories too large for participants to assign all possible exemplars to appropriate release conditions and nevertheless found priming by novel primes (Finkbeiner \& Friedman, 2011), so in terms of forced-choice priming situations at least the notion of implicit classifications remains feasible.

The third way that masked novel primes could influence responses is by automatically spreading activation within their semantic network. Unlike the implicit classification account, 
where masked primes can influence target processing only when they belong to task-defined response categories, the spreading activation account predicts that all stimuli (whether consciously or nonconsciously perceived) are automatically processed up to a semantic level. Crucially, masked primes are believed to automatically spread activation within their semantic network, facilitating the processing of subsequent targets when these belong to the same semantic network. For example, when the target "CAT" is preceded by the related prime "DOG," activation of the prime's semantic representation would activate the target's semantic representation, thus facilitating semantic access to the target (Collins \& Loftus, 1975; Neely, 1976; Posner \& Snyder, 1975; see Neely, 1991, for a review). In the number classification task, the primes 2 and 3 would automatically activate closely related concepts 1 and 4, facilitating processing of these stimuli should they appear as targets. The "automaticity" of spreading activation theories has certainly been questioned, with several studies demonstrating that a masked prime's influence over target-elicited responses depends on its task-relevance (Bodner \& Dypvik, 2005; de Wit \& Kinoshita, 2015; Klinger, Burton, \& Pitts, 2000; Kunde, Kiesel, \& Hoffmann, 2003). Interestingly, it has been proposed that activation within a prime's semantic network might also trigger its categoryassigned response (Wentura, 1999); this added mechanism would account for why, in the number classification task, the prime 4 appears to facilitate responses when the subsequent target is a 2 (belonging in the same category) but not a 6 (belonging in the opposite category).

The present study is concerned primarily with understanding the mechanisms underlying free-choice priming. It was designed to answer two questions: first, can free-choice priming emerge using a task that employs stimulus categories too large for action-triggers to operate? Second, does a masked prime's ability to bias free-choice decisions rely on implicit classifications, or does it depend on its ability to automatically spread activation in its semantic network? To address these questions participants were asked to categorise targets as people or animals by making fixed (left vs. right) responses, and to freely chose among the two response alternatives when presented with a free-choice target. All targets were preceded by a masked animal or person prime that either appeared as a target during the experiment, or was a novel category exemplar. Importantly, while primes and targets within the person category possessed response congruency (in that both were associated with the same category-assigned response), primes and targets within the animal category could also possess a pre-existing semantic relationship. For instance, a masked "lion" prime might automatically activate semantically related concepts "elephant" or "tiger" and thus facilitate semantic access to these stimuli should they appear as targets. A masked "person" prime, on the other hand, has no avenue through which to activate a semantic representation of any other particular person; these primes and targets are related only in as much as they both activate the same response.

If action-triggers underpin free-choice priming, masked primes should not influence free choice responses in the present task. In contrast, if free-choice priming emerges due to implicit classifications, both animal and person primes should bias free-choice responses, and they should do so equally often. Finally, if free-choice priming emerges due to spreading activation within the prime's semantic network, the proportion of prime-congruent free-choice responses should exceed chance-levels only following animal primes because these (and not person primes) have the potential to activate a myriad of related concepts within their semantic network.

\section{Method}

\section{Participants}

Forty-four participants (15 males) aged between 18 and 35 years were recruited from the University of Queensland subject pool. All had normal or corrected to normal vision, and gave written, informed consent prior to participating in the study. The procedures were approved by the University of Queensland Human Research Ethics Committee. Five participants were excluded for making too many errors $(>20 \%)$ during fixed trials, leaving a sample of 39 (13 males, mean age 19.8 years, SD 3.2).

\section{Stimuli and Procedure}

Stimuli were presented on a 24 -inch LCD monitor with a resolution of $1920 \times 1080$ at $60 \mathrm{~Hz}$, controlled by a Dell Optiplex 9020 running 64-bit Windows. The eye-screen distance was approximately $45 \mathrm{~cm}$. Responses were executed with the index fingers of both hands by pressing the " $Z$ " and "M" keys of a standard QWERTY keyboard. A total of eight targets were used for the fixed trials, including four animals and four people (Fig. 1). Two of these targets (one animal and one person) also served as primes. Because these primes were experienced and responded to as targets throughout the experiment, they were termed "repeated primes." The stimuli were also regarded as "filler targets" to distinguish them from targets that were physically distinct from all primes. Novel primes were one person and one animal that were never displayed as targets and thus appeared only under masked conditions. The free choice-target was an arbitrary stimulus (the image of a tree). All stimuli were grey-scale and subtended a visual angle of $2.42^{\circ} \times 4.12^{\circ}$.

Details of the trial structure are depicted in Fig. 2. Participants were informed that an image would appear centrally on the screen and that their task was to categorise that image as a person or an animal by making a left or right button-press 


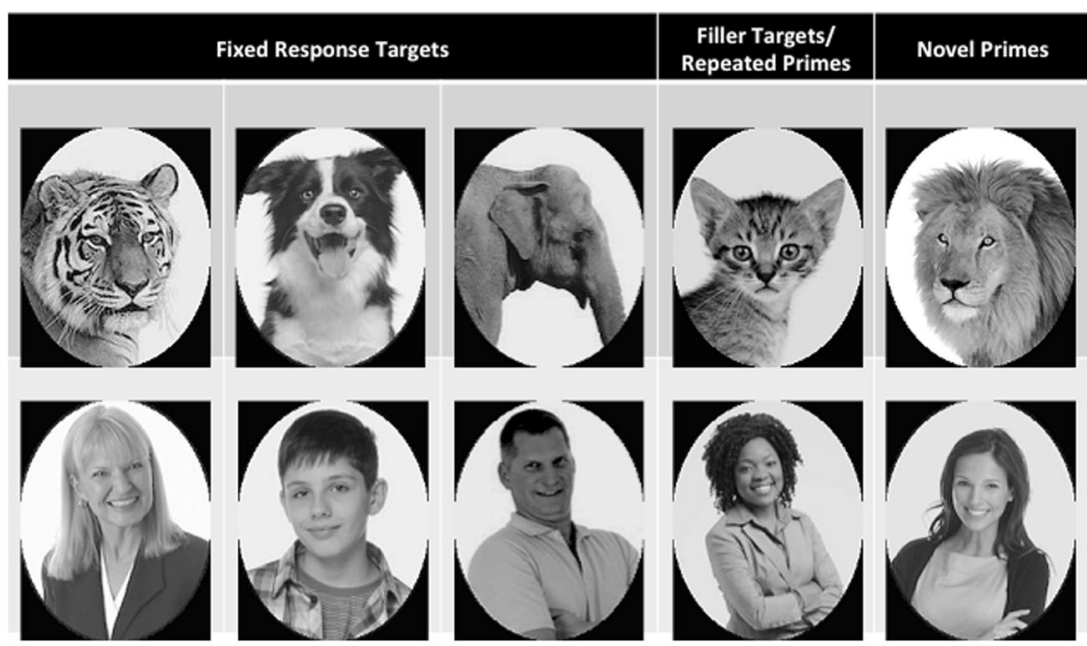

Fig. 1 Stimuli used in experiment.

response (stimulus-response mappings were counterbalanced across participants). They were instructed to freely and randomly select a left or right response when the free-choice target appeared. It was emphasized that they should attempt to perform each response roughly equally often, without following a fixed response scheme (e.g., alternating between left and right). Incorrect and late responses were indicated by a buzz and visual feedback (e.g., "WRONG" or "Too Late!").

The combination of 8 targets ( 4 animals and 4 people) and 4 primes ( 2 repeat and 2 novel) yielded 32 trials per block. Randomly intermixed among these fixed trials were 12 trials containing 3 repetitions of the free-choice target across each prime type, producing a total of 44 trials in each block. After completing two practice blocks, participants completed 616 trials spread across 14 experimental blocks. Filler targets were excluded from the analysis to ensure that the relationship between each prime and target combination was equivalent (i.e., both stimuli remaining physically distinct). As a result, 504 trials from each participant were subjected to analysis.

After completing the main experiment participants were asked whether they had noticed any stimuli apart from the masks and targets. Then, they were informed about the primes

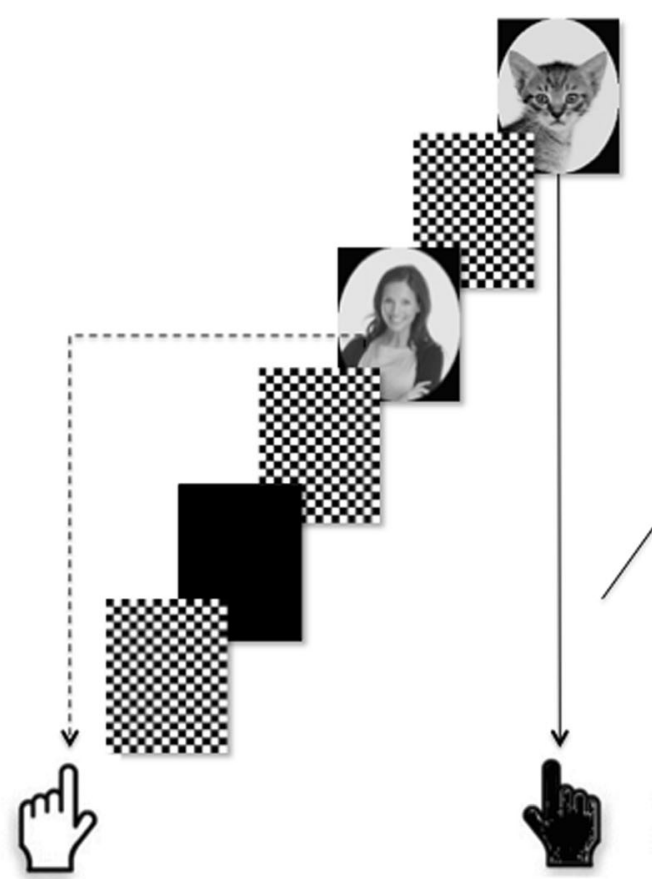

Fig. 2 Experimental task. In this example, a subject must classify a person by making a left button-press response, and an animal by making a right button-press response. The left side illustrates an incongruent fixed trial with a novel prime and a filler target. Although

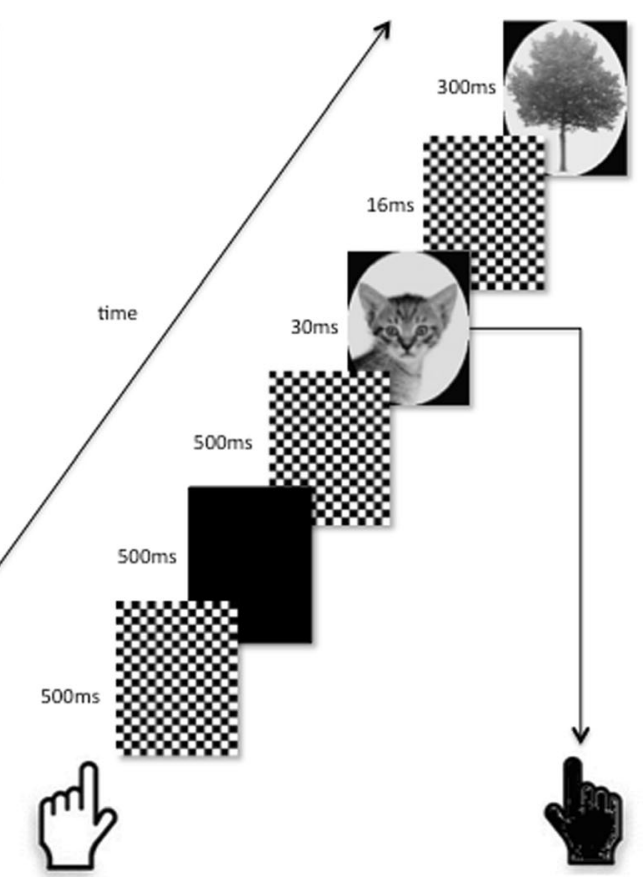

the prime never appears as a target, it affords an incongruent response. On the right is an example of a congruent free-choice trial in which the selected response was congruent with a repeated prime. Prime duration was $30 \mathrm{~ms}$; target duration was $300 \mathrm{~ms}$. 
and were specifically asked whether they had noticed their presence. Next, an objective measure of prime awareness was taken. The prime-classification block consisted of 64 fixed and 24 free-choice trials that were identical to those in the main experiment. After a response to the target was given, participants were asked to classify the prime as either a person or an animal, and to "just guess" if no prime was perceived. To calculate $d$ ', trials with a person prime were designated as the "signal only" trials, and trials with an animal prime were designated as the "noise only" trials. A prime classification of "person" was scored as a hit during signal only trials, and as a false alarm during noise only trials.

\section{Results}

\section{Fixed priming}

Reaction time (RT) and accuracy data were subjected to $2 \times 2$ $\times 2$ ANOVAs with target category (animal vs. person), primetarget congruity (whether or not the prime and target belonged to the same stimulus category), and prime type (novel vs. repeat) as within-subjects factors. The RT analysis revealed a significant main effect of target category $(F(1,38)=25.48, p<$ $0.01, d=0.29)$, with overall faster RTs to person compared with animal targets $(M=504.46 \mathrm{~ms}, S E=4.79$ vs. $M=520.95$ $\mathrm{ms}, S E=4.56$, respectively), and a main effect of prime-target congruity $(F(1,38)=12.26, p<0.01, d=0.10)$, with faster RTs when the prime and target belonged to the same compared with the opposite stimulus category $(M=509.70 \mathrm{~ms}, S E=$ 4.76 vs. $M=515.71 \mathrm{~ms}, S E=4.58$, respectively; Fig. 3). There were no other main effects or interactions.
The accuracy analysis revealed a significant main effect of target category $(F(1,38)=7.11, p<0.05, d=0.36)$, with higher accuracy rates to person targets $(94.2 \%)$ compared with animal target $(92 \%)$. Furthermore, there was a target category x prime type interaction $(F(1,38)=7.83, p<0.05, d=0.20)$. Paired-samples $t$ tests revealed that accuracy rates were marginally higher to person targets following novel compared with repeat primes $(94.9 \%$ and $93.5 \%$ respectively, $p<0.05$ with Bonferroni correction).

\section{Free-choice priming}

One subject did not follow instructions regarding the free choice trials (they responded with only one key) and were thus excluded from this analysis. However, because they were included in the analysis of RTs, they were retained for the analysis of prime visibility. Free-choice RTs were analysed using a $2 \times 2 \times 2$ ANOVA with prime category (animal vs. person), response congruity (whether the response was congruent or incongruent with the preceding prime), and prime type (novel vs. repeat) as within-subjects factors. A significant interaction was found between prime category and response congruity $(F(1,37)=6.08, p<0.05, d=0.23)$. Paired-samples $t$-tests indicated that RTs were faster when free-choice responses were congruent $(M=527.61, S E=2.65)$ versus incongruent $(M=549.06, S E=3.10)$ with an animal prime $(t(37)=2.94, p$ $<0.05$ with Bonferroni correction; Fig. 4). Free-choice responses congruent versus incongruent with person primes did not differ in terms of RTs.

These results indicate that prime-congruent responses were performed faster than prime-incongruent responses following presentation of an animal prime, but the major focus of this

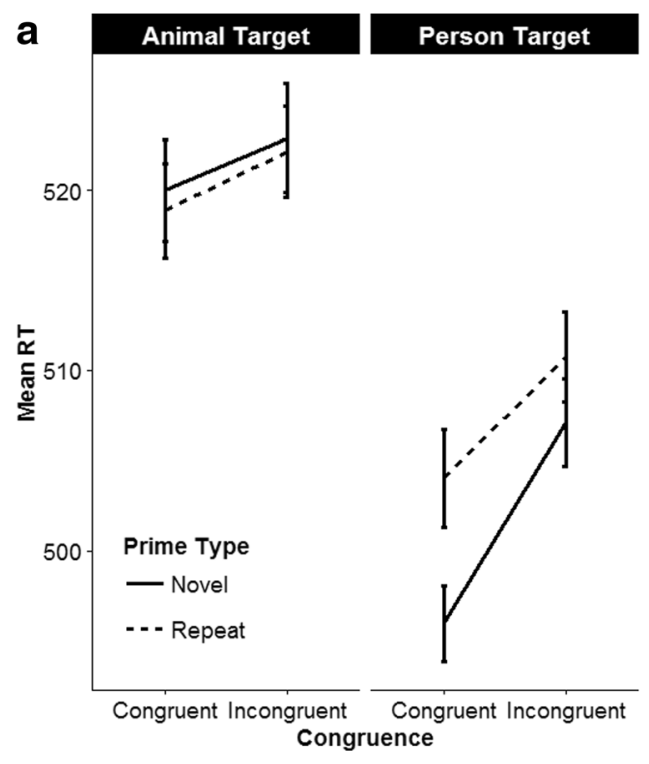

Fig. 3 Fixed priming. Mean reaction times (a) and accuracy rates (b) to animal and person targets during congruent trials (both the prime and target belonged to the same stimulus category) and incongruent trials

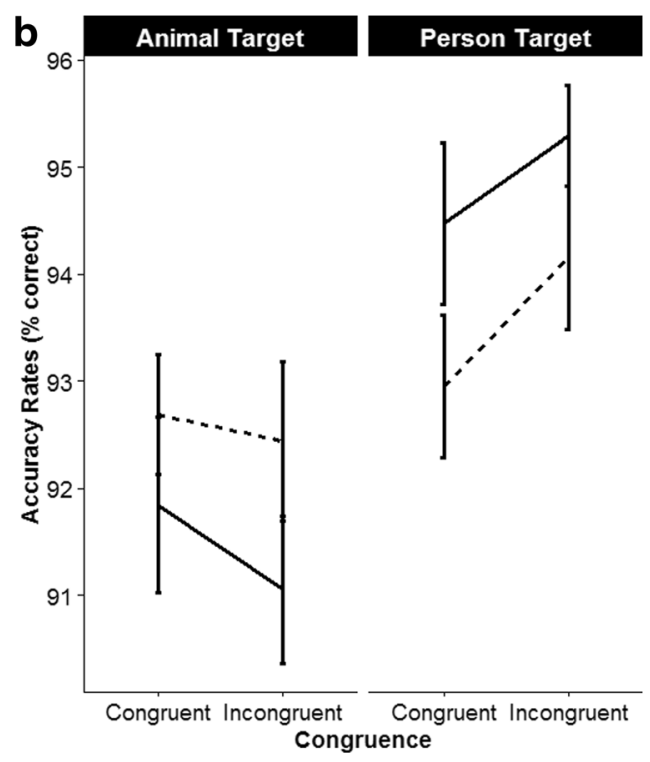

(the prime and target belonged to different stimulus categories), separately for novel and repeat primes. The bars represent the standard error of the mean $(S E M)$. 


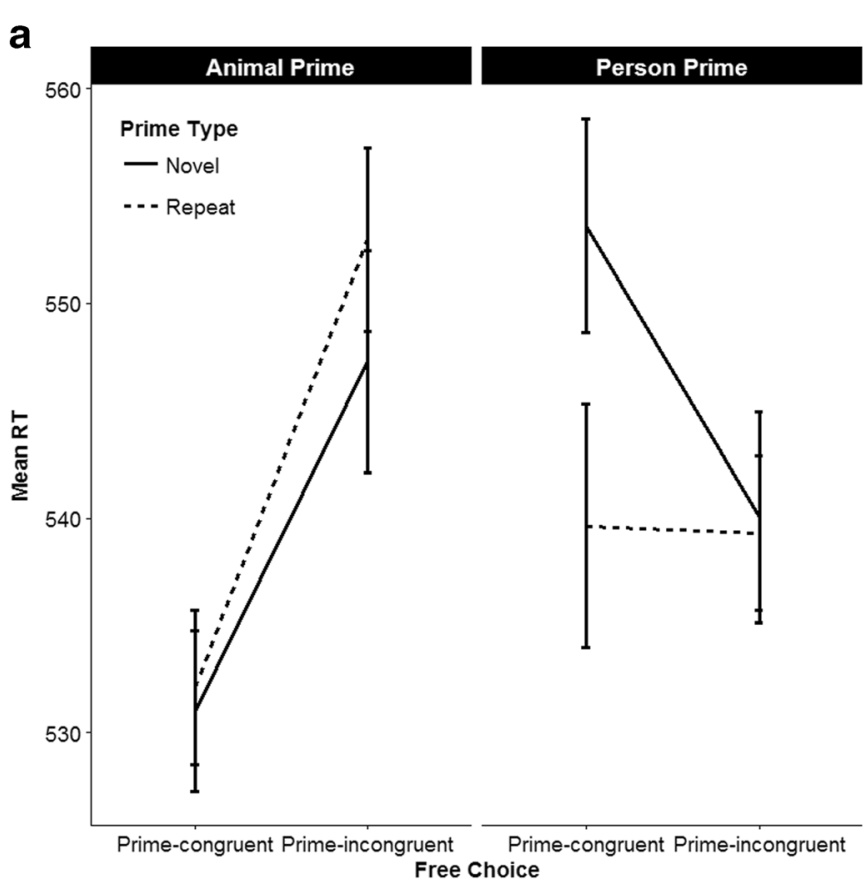

Fig. 4 Free-choice priming. Mean reaction times (a) to the free-choice target following animal (left) and person (right) primes for primecongruent choices (where response choices corresponded with the preceding prime) and prime-incongruent choices (where response

analysis was the bias produced by masked primes on response selection. Of particular interest was the relative influence of the two prime categories (animals vs. people), and prime types (novel vs. repeat) on participants' free choice decisions. The measure was the percentage of responses that were congruent with the masked prime. There was a main effect of prime category $(F(1,37)=6.03, p<0.05, d=0.75)$; participants made more prime-congruent responses following animal primes $(57 \%)$ than following person primes $(44 \%)$. There were no other main effects or interactions.

\section{Prime visibility}

On the prime awareness question, no participants reported awareness of the primes. Once informed about the true nature of the trial sequence, three participants reported noticing "something" between the masks on at least one trial. On the prime classification task, participants' ability to discriminate the prime was minimal, producing a $d$ ' of 0.04 which did not differ significantly from $0(p=0.20)$. The overall mean hit and false alarm rates were low (25\% and $24 \%$ respectively), which could indicate a potential bias in participants' responses and hence reduce the interpretability of the $d$ ' score. As such the $d$ ' analysis was supplemented with an analysis of individual accuracy rates (i.e., \% correct prime classifications) against a population mean of 0.5 . This analysis revealed that the overall percentage of correct prime classifications $(51.1 \%)$ did not differ significantly from chance $(p=0.28)$. To explore whether

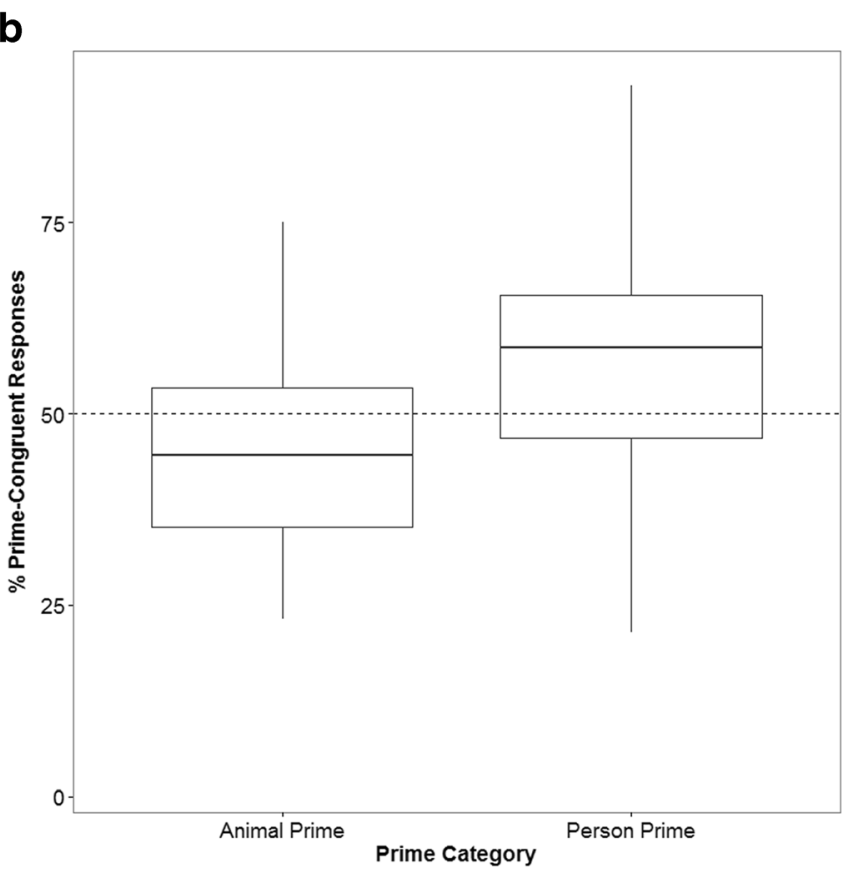

choices did not correspond with the preceding prime), separately for novel and repeat primes. Percentage of free-choice responses (b) congruent with an animal (left) and a person (right) prime. All bars represent the standard error of the mean (SEM).

prime awareness was influenced by prime category (animal vs. person), prime type (novel vs. repeat) or prime-target congruity (both stimuli belonging to the same vs. the opposite stimulus category), these factors were entered into a $2 \times 2 \times$ 2 ANOVA with individual prime classification scores as the dependent measure. A main effect of prime-target congruity was found, with a lower percentage of correct prime classifications when the prime and target belonged to the same category $(45.9 \%)$ compared with the opposite category $(56.7 \%$; $F(1,38)=8.37, p<0.01, d=0.6)$. This finding suggests that non-conscious processes associated with responding to the target could have modulated prime classification performance. To obtain a measure of prime awareness that was not influenced by prime-target congruity, a one-sample $t$ test was performed over trials in which the prime was followed by the free-choice target. Prime classification accuracy in these trials was found to be at chance $(52.8 \% ; p=0.10)$.

It was nevertheless necessary to establish that the priming effects observed on fixed trials were not driven by a subset of participants with extreme prime-classification scores. To this end, the fixed-choice analysis on RTs was repeated, this time with individual prime-classification scores included as covariates. The ANCOVA revealed that the main effects reported in the fixed-choice RT analysis were unaltered by controlling for prime-classification scores. While these findings do not rule out the influence of conscious perception, they suggest that the results reported in this experiment were not related to the degree participants were able to classify the primes. 


\section{Discussion}

In free-choice priming paradigms, participants are asked to choose which of two response alternatives they wish to make when presented with a free-choice target. More often than not they select the response alternative that is congruent (versus incongruent) with a masked prime. Recently, Ocampo (2015) used a number classification task to demonstrate that masked primes can bias free-choice responses even in the absence of automatized S-R links, suggesting that response selections were influenced by a nonconscious appreciation of the masked prime's meaning. Whether free-choice priming was caused by the implicit classification of masked primes versus spreading activation within their semantic network, however, remained uncertain. I report the results of an experiment in which participants were asked to categorise targets as either people or animals. These large stimulus categories were chosen to rule out action-trigger accounts of priming (Kiesel et al., 2007). Free-choice targets were randomly intermixed among fixed-choice targets, and all were preceded by a masked prime that either doubled as a (fixed-choice) target or was a novel category exemplar. Crucially, while both animal and person primes could be subjected to implicit classifications, only animal primes were able to trigger spreading activation within their semantic network.

On fixed-choice trials, responses to both animal and person targets were faster when masked primes belonged to the same versus the opposite stimulus category, regardless of whether the prime had appeared as a target or not. This result confirmed that both repeat and novel masked primes were effective in modulating target-elicited responses. The issue at stake, however, was whether the proportion of prime-congruent freechoice responses differed depending on whether the masked prime was an animal or a person. The results were in line with spreading activation accounts; participants were more likely to select a prime-congruent than a prime-incongruent response following an animal prime, but not a person prime. Freechoice responses congruent with a preceding animal prime were also performed faster compared with incongruent responses. Once again, the effects were not modulated by whether or not the prime had appeared as a target, confirming that S-R associations alone could not account for these results.

If priming emerged because masked primes automatically spread activation in their semantic network, how did person primes (which cannot generate such spreading activation) produce priming during fixed-choice trials? One possibility is that both animal and person primes were processed up to a semantic level, but spreading activation across related concepts occurred only for animal primes, allowing these stimuli to bias response selections on free-choice trials. If we also assume that semantic-level processing of a masked prime automatically activates its category-assigned response (Wentura, 1999), person primes would be able to facilitate responses to congruent (person) targets and interfere with responses to incongruent (animal) targets. An alternative possibility is that the critical role of spreading activation during free-choice trials does not preclude implicit classification processes from taking place; both implicit classification and automatic spreading activation might occur in parallel, the former producing priming on fixed-choice trials and the latter on freechoice trials. On this account, implicit classifications would activate a masked prime's category-assigned response, but they would not determine if the response would be executed during free-choice trials; this would remain contingent on spreading activation processes. Further investigations are needed to distinguish between these two possibilities.

Previous studies employing the arrow classification task have demonstrated free-choice priming driven primarily by automatized S-R links (i.e., in the absence of semantic-level processing; Bodner \& Mulji, 2010; Mattler \& Palmer, 2012; Kiesel et al., 2006; Klapp \& Hinkley, 2002; Schlaghecken \& Eimer, 2004). However, if S-R links can produce free-choice priming under certain circumstances, then why did repeat primes in the person category (for which S-R links could have been established) fail to produce the effect? Several methodological differences between this study and those that employed the arrow classification task might help to shed light on this discrepancy. Most notably, previous experiments employed only two fixed-choice targets (typically a left and right arrow) that also doubled as masked primes. Left and right arrows were associated with left and right button-press responses, and throughout the experiment these associations were automatized and continuously reinforced. In contrast, participants in this experiment were shown a total of eight targets, only two of which doubled as masked primes (one animal and one person). These so-called "filler targets" represented only $\sim 18 \%$ of the experimental trials. One could argue, therefore, that the S-R associations established for these stimuli were too weak to produce a bias to participants' free-choice selections.

No study is perfect and this one is no exception. Whether the present task required semantic-level processing or whether the classification of pictures of people and animals was predominantly a perceptual task remains to be determined. Furthermore, it seems that prime type had a different impact on responses to animal and person targets. Specifically, accuracy rates to person targets were higher when preceded by novel compared with repeat primes whereas animal targets were classified with equal accuracy under both novel and repeat prime conditions. Although numerically small (1.4\%), the effect in question was significant and therefore warrants an interpretation. Previous findings have shown that stimuli characterised by distinctive yet task-relevant perceptual features benefit from enhanced saliency and boosted perceptual processing (Corbetta \& Shulman, 2002). Masked novel primes are in possession of both these characteristics in that 
they are physically distinct category exemplars belonging to participants' currently active task set. If additional attentional resources were allocated to novel primes, this could have enhanced the processing of subsequent targets. The problem with this interpretation is that response facilitation did not emerge for animal targets, where accuracy rates were unaltered by prime type. Exactly why this is remains an open question, but one possibility is that the enhanced perceptual processing generated by novel primes is relatively short-lived and would dissipate at the longer response latencies observed for animal targets.

To conclude, in the context of a semantic categorisation experiment the influence of masked primes on free choices hinged on their ability to trigger spreading activation within their semantic network. It remains the task of future research to replicate this effect using different stimulus categories that differ only in terms of membership in a network of semantically related concepts. This would establish that similar to fixed-choice priming, free-choice priming can be underpinned by a number of different processes including, at least, stimulus-response associations and spreading activation within the prime's semantic network.

\section{References}

Bodner, G. E., \& Dypvik, A. T. (2005). Masked priming of number judgments depends on prime validity and task. Memory \& Cognition, 33(1), 29-47.

Bodner, G. E., \& Mulji, R. (2010). Prime proportion affects masked priming of fixed and free-choice responses. Experimental Psychology, 57(5), 360.

Collins, A. M., \& Loftus, E. F. (1975). A spreading-activation theory of semantic processing. Psychological Review, 82(6), 407.

Corbetta, M., \& Shulman, G. L. (2002). Control of goal-directed and stimulus-driven attention in the brain. Nature Reviews Neuroscience, 3(3), 201-215.

de Wit, B., \& Kinoshita, S. (2015). The masked semantic priming effect is task dependent: Reconsidering the automatic spreading activation process. Journal of Experimental Psychology: Learning, Memory, and Cognition, 41(4), 1062.

Dehaene, S., Naccache, L., Le Clec'H, G., Koechlin, E., Mueller, M., Dehaene-Lambertz, G., \& Le Bihan, D. (1998). Imaging unconscious semantic priming. Nature, 395(6702), 597-600. https:// doi.org/10.1038/26967

Finkbeiner, M., \& Friedman, J. (2011). The flexibility of nonconsciously deployed cognitive processes: evidence from masked congruence priming. PloS One, 6(2), e17095.
Forster, K. I., \& Davis, C. (1984). Repetition priming and frequency attenuation in lexical access. Journal of Experimental Psychology: Learning, Memory, and Cognition, 10(4), 680.

Kiesel, A., Kunde, W., \& Hoffmann, J. (2007). Mechanisms of subliminal response priming. Advances in Cognitive Psychology, 3(1-2), 307315. https://doi.org/10.2478/v10053-008-0032-1

Kiesel, A., Wagener, A., Kunde, W., Hoffmann, J., Fallgatter, A. J., \& Stöcker, C. (2006). Unconscious manipulation of free choice in humans. Consciousness and Cognition, 15(2), 397-408. https:// doi.org/10.1016/j.concog.2005.10.002

Klapp, S. T. (2015). One version of direct response priming requires automatization of the relevant associations but not awareness of the prime. Consciousness and Cognition, 34, 163-175.

Klapp, S. T., \& Haas, B. W. (2005). Nonconscious influence of masked stimuli on response selection is limited to concrete stimulus-response associations. Journal of Experimental Psychology: Human Perception and Performance, 31(1), 193.

Klapp, S. T., \& Hinkley, L. B. (2002). The negative compatibility effect: unconscious inhibition influences reaction time and response selection. Journal of Experimental Psychology: General, 131(2), 255.

Klinger, M. R., Burton, P. C., \& Pitts, G. S. (2000). Mechanisms of unconscious priming: I. Response competition, not spreading activation. Journal of Experimental Psychology: Learning, Memory, and Cognition, 26(2), 441.

Kunde, W., Kiesel, A., \& Hoffmann, J. (2003). Conscious control over the content of unconscious cognition. Cognition, 88(2), 223-242. https://doi.org/10.1016/S0010-0277(03)00023-4

Mattler, U., \& Palmer, S. (2012). Time course of free-choice priming effects explained by a simple accumulator model. Cognition, 123(3), 347-360. https://doi.org/10.1016/j.cognition.2012.03.002

Naccache, L., \& Dehaene, S. (2001). Unconscious semantic priming extends to novel unseen stimuli. Cognition, 80(3), 215-229.

Neely, J. H. (1976). Semantic priming and retrieval from lexical memory: Evidence for facilitatory and inhibitory processes. Memory \& Cognition, 4(5), 648-654.

Neely, J. H. (1991). Semantic priming effects in visual word recognition: A selective review of current findings and theories. Basic Processes in Reading: Visual Word Recognition, 11, 264-336.

Ocampo, B. (2015). Unconscious manipulation of free choice by novel primes. Consciousness and Cognition, 34, 4-9.

Posner, M. I., \& Snyder, C. (n.d.). R.(1975).“Attention and cognitive control," (pp. 55-85). Presented at the Information processing and cognition: The Loyola symposium.

Reynvoet, B., Gevers, W., \& Caessens, B. (2005). Unconscious primes activate motor codes through semantics. Journal of Experimental Psychology: Learning, Memory, and Cognition, 31(5), 991

Schlaghecken, F., \& Eimer, M. (2004). Masked prime stimuli can bias "free" choices between response alternatives. Psychonomic Bulletin \& Review, 11(3), 463-468. https://doi.org/10.3758/BF03196596

Wentura, D. (1999). Activation and inhibition of affective information: For negative priming in the evaluation task. Cognition \& Emotion, 13(1), 65-91. 